(4) Ein ideales Maß von Gesetzgebungsdauer existiert nicht und lässt sich abstrakt auch nicht festlegen. Es trifft zu, dass Gesetzgebungsverfahren in Deutschland vergleichsweise lange dauern. In Großbritannien benötigten die in der Session 2004/05 verabschiedeten Gesetze rund 113 Tage; in Bayern brauchte ein Gesetz von der Einbringung bis zur Verkündung in der 14. Wahlperiode (1998 bis 2003) circa 137 Tage. ${ }^{22}$ Doch taugen solche Vergleiche keineswegs dazu, normative Standards zu bilden. Kurze oder kürzere Gesetzgebungsverfahren als in Deutschland sind nicht per se die besseren.

22 Eigene Auszählungen auf Grundlage von: Bayerischer Landtag, Tätigkeitsbericht über die 14. Wahlperiode 1998/2003, München, http://www.landtag-bayern.de/pdf_internet/Taetigkeitsbericht_14WP.pdf (Stand: 13. Mai 2006) sowie dem Sessional Information Digest des House of Commons, http://www.publications.parliament.uk/pa/cm/cmsid.htm (Stand 10. April 2006). Das Gesetzgebungsverfahren in Großbritannien beginnt mit der ersten Lesung und endet mit dem Royal Assent, in Bayern wird das Verfahren begrenzt durch den Tag der Einbringung eines Gesetzentwurfs in den Landtag (Drucksachendatum) und dem Tag der Verkündung des Gesetzes.

\title{
Private Bills in angelsächsischen Regierungssystemen: Legitimitätsressource oder Unterlaufen der Gewaltenteilung?
}

\section{Thomas Krumm}

Anders als in der Rechtswissenschaft ${ }^{1}$ ist das in den angelsächsisch geprägten Regierungssystemen verbreitete Gesetzgebungsverfahren der Private Bills von der deutschen Politikwissenschaft bislang fast überhaupt nicht wahrgenommen worden. Private Bills eröffnen einzelnen privaten Akteuren und Interessen die Möglichkeit, Gesetzgebung durch einzelne Abgeordnete, die als parlamentarische „Paten“ fungieren, zu initiieren, die sich explizit zugunsten dieser privaten Akteure auswirkt. Solche Private Bills sind nicht zu verwechseln mit Private Members' Bills, dem Recht einzelner Abgeordneter, im Bereich der allgemeinen Public Bills initiativ zu werden. Private Bills können, im Unterschied zu den Public Bills, einzelnen Individuen bestimmte Vorteile gewähren wie etwa Ausnahmen von den allgemeinen Gesetzen. In der Bundesrepublik Deutschland würde man diesen Gesetzestyp als Einzelfall- und Maßnahmengesetz bezeichnen. Absicht der folgenden Untersuchung ist eine vergleichende Einführung in die „private“ Gesetzgebung in Großbritannien, Schottland, den USA und Kanada sowie eine Gegenüberstellung mit den in der Bundesrepublik Deutschland stark eingeschränkten, aber nicht grundsätzlich verbotenen Einzelfall- beziehungsweise Maßnahmengesetzen.

1 Grundlegend nach wie vor Julius Hatschek, Englisches Staatsrecht, Tübingen 1905, Band 1, S. $120 \mathrm{ff}$. und S. 503 ff., Band 2, S. 376 f. und passim. 
Auffällig ist zunächst die Nichtrezeption des Themas „private legislation“ durch die bundesdeutsche Parlamentarismusforschung; diese selektive Blindheit kann bedeuten, dass auf Grund des Nichtvorhandenseins einer entsprechenden Institution im bundesdeutschen wie in kontinentaleuropäischen Regierungssystemen eine Sensibilität für die damit verbundene verfassungs- und demokratietheoretische Relevanz des Themas fehlt. Hier ist sicherlich auch zu erwähnen, dass die Vorstellung, natürliche oder juristische Privatpersonen könnten legislatorisch initiativ werden, mit dem kontinentaleuropäischen Parlamentsverständnis nicht zusammen geht. Außerdem ist in der Bundesrepublik das Recht, einen Gesetzentwurf einzubringen, an ein Abgeordnetenquorum ${ }^{2}$ gebunden und steht nicht einzelnen Abgeordneten zu, wie das bei der Form der Private Members' Bills ${ }^{3}$ in den angelsächsischen Ländern der Fall ist, die die Initiativfunktion gerade auch für parlamentarische „Hinterbänkler“ öffnet, wenn auch mit letztlich geringen Erfolgsaussichten.

\section{Private Bills als Legitimationsressource?}

Das Private Bills-Verfahren hat sich im Vereinigten Königreich entwickelt, wo es dem Zweck diente, Abhilfe bei spezifischen Problemen zu schaffen oder einzelnen Personen bestimmte Vorteile zu gewähren, die durch Public Law beziehungsweise Common Law nicht möglich waren. So waren etwa Ehescheidungen in Kanada bis zur Einführung eines entsprechenden allgemeinen Gesetzes 1968 nur im Private Bills-Verfahren möglich. Rechtsgeschichtlich eng verwandt sind die im Vereinigten Königreich 1870 verbotenen „Bills of Attainder“, bei denen Krone und Parlament die Möglichkeit hatten, per Gesetzesbeschluss Personen ohne ein Gerichtsverfahren zu verurteilen. ${ }^{4}$ Für die USA liegen Nachweise bis 1817, für Kanada bis 1867 und für Großbritannien bis 1801 auf der Parlamentshomepage vor. Für alle Länder gilt, dass Private Bills ihre Hochphase im 19. Jahrhundert bei der Planung und Durchführung von Infrastrukturmaßnahmen hatten, mit unterschiedlichen Akzenten aber bis in die Gegenwart zur Regulierung privater Interessen vor allem im Infra-

2 Art. 76 Abs. 1 GG nennt als initiativberechtigt die Bundesregierung, die „Mitte des Bundestages“ und den Bundesrat. Die „Mitte des Bundestages“ umfasst dabei mindestens fünf Prozent der Abgeordneten oder eine Fraktion.

$3 \mathrm{Zu}$ einer Verwechslung von Private Members' Bills und Private Bills kommt es etwa bei Jürgen Hartmann, Westliche Regierungssysteme, Wiesbaden 2005, S. 70, wenn er schreibt, dass sich Private Members' Bills „stets auf Gesetzesbeschlüsse für Einzelpersonen, Gemeinden oder öffentliche Körperschaften“ bezögen. „Es handelt sich um Einzelfallgesetze (private laws), denen neben den allgemeinen Gesetzen (public laws) keine große Bedeutung zukommt." Vielmehr gibt es für Private Members' Bills keine Einschränkung auf Einzelfallgesetze oder bestimmte Regelungsmaterien. Das „Gegenstück“ von Private Bills sind Public Bills, von Private Members’ Bills dagegen Government Bills.

4 Für Deutschland lag eine vergleichbare Einrichtung im königlichen „Machtspruch“ vor, verfassungsgeschichtlich relevant geworden im so genannten Müller-Arnold-Fall von Friedrich II. Im „Müller-Arnold-Fall“ (1770 bis 1779) ließ Friedrich II. gleich mehrere Richterkollegien verhaften und ihre Urteile aufheben, weil sie einem Müller, der dagegen geklagt hatte, dass man dem Fluss an seiner Mühle das Wasser abgegraben hatte, so dass er nicht mehr genug erwirtschaften konnte, um den Erbzins an den Landsherren zu zahlen, nicht „Gerechtigkeit“ widerfahren ließen, sondern lediglich die geltenden Gesetze angewandt hatten, vgl. Werner Frotscher / Bodo Pieroth, Verfassungsgeschichte, München 2002, Rn. $131 \mathrm{ff}$. 
strukturbereich, in den USA aber auch mit einem Schwerpunkt in Immigrationsfragen, behalten haben.

Private Bills unterscheiden sich von Public Bills durch substanziell verschiedene Verfahren in der parlamentarischen Behandlung, durch ein spezielles Initiativrecht, bei dem ein parlamentarischer „Promotor“ oder „Agent“ für einen privaten Klienten aktiv wird und schließlich durch die faktischen Besonderheiten der Regelungsmaterie. Private Bills umfassen Maßnahmen, die primär im privaten Interesse des Initiators liegen und gegen die potenziell betroffene Dritte Einspruch erheben können mit dem Hinweis, durch die Maßnahme in ihren eigenen Interessen betroffen zu sein. Die Bezeichnung Private Bill sowie der private Klient sollten nicht darüber hinwegtäuschen, dass es sich um ein vollständig parlamentarisches Verfahren handelt, an dessen Durchführung ausschließlich Parlamentarier beteiligt sind. Das Parlament bleibt in der Rolle des Gesetzgebers, wenngleich diese Rolle viel stärker auf die Schlichtung widerstreitender privater Interessen konzentriert ist. Dadurch gewinnt die Legislation von Private Bills einen Doppelcharakter zwischen einerseits parlamentarischem Verfahren und andererseits einem quasi-gerichtlichen Prozess. ${ }^{5}$ Mit diesem Doppelcharakter bewegen sich Private Bills auch an einer bislang weitgehend unbeachteten Schnittstelle von Legislative und Judikative. Während zum Beispiel die britischen Verfassungsreformen der New Labour-Regierung mit dem Hinweis auf die weitere Differenzierung der drei Gewalten, in deren Hauptschnittpunkt sich bislang der Lord Chancellor bewegte, begründet worden sind, ist die Institution der Private Bills unter dem Gesichtspunkt der „differentiated polity“6, der Weiterentwicklung der Gewaltenteilung, beispielsweise durch die Abschaffung des Amts des Lord Chancellors und der Einrichtung eines Supreme Courts, bislang nicht thematisiert worden. Vielmehr wurde im Zuge der Devolution bei der Einrichtung des Schottischen Parlaments private Gesetzgebung auch in der Region ermöglicht. Das Beispiel Schottland illustriert, wie sehr Private Bills kennzeichnend für vom britischen Westminister-Modell beeinflusste Systeme sind. Das Initiativrecht für private Interessen findet sich aber nicht nur in parlamentarischen Demokratien des Westminister-Typs wie Großbritannien, Irland, Kanada, Neuseeland und Australien, sondern auch im präsidentiellen System der USA.

Neben dem quasi-gerichtlichen Charakter, den Private Bills einnehmen können, spielt auch die administrative Dimension in dieses Gesetzgebungsverfahren hinein. Dies lässt sich im Folgenden gut an Beispielen aus Großbritannien und den USA erläutern, in denen in den letzten Jahrzehnten entsprechende Regelungskompetenzen an Ministerien und Behörden übertragen wurden, was zu einem Rückgang privater Gesetzgebungsinitiativen geführt hat. Diese Kompetenzverlagerung zugunsten der Verwaltung macht deutlich, dass Private Bills nach kontinentaleuropäischem Verständnis von Gewaltenteilung auch quer zur Differenzierung von Legislative und Exekutive liegen. Nach bundesdeutschem Verständnis ist der Bundestag für die Gesetze und die Ermächtigung durch Gesetz zuständig, die Bundesregierung beziehungsweise die Verwaltung für die Details der Ausführung durch rangniede-

5 Vgl. www.scottish.parliament.uk/business/bills/privateBills.htm, 6. März 2006.

6 Mit dem von R.A.W. Rhodes, Understanding Governance, Buckingham 1997, geprägten Begriff der differentiated polity lassen sich die umfangreichen Verfassungs- und Justizreformen der Regierung Tony Blair, die auf eine Stärkung der Gewaltenteilung und die Einführung neuer Governance-Konzepte gezielt haben, umfassend analysieren. 
re Rechtsverordnungen. ${ }^{7}$ Dass sich diese idealtypische Trennung in der Regierungspraxis nicht immer klar aufrecht erhalten lässt und es auch im bundesdeutschen Regierungssystem zu einer quasi „übergreifenden“ Legislativtätigkeit kommen kann, wird im vierten Abschnitt erörtert.

Geleitet wird die folgende Dokumentation von der Frage, ob Private Bills eine besondere, eventuell nachahmenswerte Legitimationsressource in angelsächsischen Regierungssystemen darstellen und ob ihre Rückläufigkeit in den letzten Dekaden folglich als Legitimationsverlust beziehungsweise als eine Entpolitisierung des Regierens gewertet werden kann. Vermutlich resultiert, so lässt sich als Ausgangsthese formulieren, der unterschiedliche Stellenwert von Private Bills beziehungsweise Einzelfall- und Maßnahmengesetzen im internationalen Rechtskreis aus unterschiedlichen Ausformungen und Verständnissen von Gewaltenteilung und parlamentarischer Aufgabenstellung. Private Bills wären dann eine von der bundesdeutschen Parlamentarismusforschung bislang weitgehend übersehene Besonderheit des Westminster-Modells und seiner speziellen politischen Kultur, die zugleich aber auch Legitimititätsfragen der bundesdeutschen „Verschlossenheit“ des Gesetzgebungsverfahrens aufwerfen würde, wie sie etwa im Abgeordnetenquorum und auch gegenüber Volksbegehren zum Ausdruck kommt.

\section{Großbritannien und Schottland}

\subsection{Private Bills in Großbritannien}

In Großbritannien sind Private Bills im 19. Jahrhundert sehr gebräuchlich gewesen. ${ }^{8}$ Sie wurden damals insbesondere für die Errichtung von Docks und Häfen sowie von Eisenbahn-, Gas- und Wasserinfrastruktur eingesetzt, während sich gegenwärtig nur noch wenige Private Bills finden. In den Sitzungsperioden 2004/05 und 2005/06 wurden jeweils sechs Private Bills im Parlament verhandelt. ${ }^{9}$ Dieser Rückgang hängt auch mit einer Reform des Systems Anfang der 90er Jahre zusammen, die zu einer Ausgliederung von Maßnahmen im Bereich der Infrastruktur geführt hat. 1987/88 kam es zu einer Komplettrevision des Systems privater Legislation durch ein Joint Select Committee. Auf Initiative des Ausschusses kam 1992 der „Transport and Works Act“ zustande, der „works bills“ aus dem Regelungsbereich von Private Bills ausnahm. Das neue Verfahren hat Maßnahmen im Straßenbahnund Eisenbahnbau, des Brücken-, Kanal- und Hafenbaus an die Verwaltung delegiert, wo sie nach einem System von Orders nach dem „Transport and Works Act“ bearbeitet werden. Wie bei Private Bills müssen auch hier Pläne hinterlegt und ein Umweltgutachten erstellt werden, es finden Anhörungen statt und es gibt Einspruchsmöglichkeiten. Allerdings finden diese Verfahren in der Regel nicht mehr unter parlamentarischer Kontrolle statt. ${ }^{10}$ Entsprechende Baumaßnahmen sind quasi von der Legislative in die Exekutive verlagert worden. Gegenwärtig sind Private Bills insbesondere bei Einwanderungsfragen und Anspruchs- beziehungsweise Forderungssachen gebräuchlich.

7 Nach Art. 80 Abs. 1 S. 2 GG müssen „Inhalt, Zweck und Ausmaß der erteilten Ermächtigung im Gesetz bestimmt werden".

8 Zur einschlägigen Entwicklung dort eingehend Julius Hatschek, a.a.O., Band 1, S. 503 ff.

9 Vgl. www.publications.parliament.uk/pa/cm/cmwib/pri.htm, 6. März 2006.

10 House of Commons Information Office, Private Bills, Factsheet L4, London 2005, S. 7. 
Ihre Einbringung erfolgt durch einen parlamentarischen Repräsentanten zugunsten des begünstigten Privaten, des „Sponsors“ einer solchen Gesetzesinitiative. ${ }^{11}$ Private Bills suchen für ihre Initiatoren Rechte und Befugnisse, die über das hinausgehen, was in Public Bills und Common Law geregelt ist. Der private oder auch kommunale Initiator benötigt dazu „Parliamentary Agents“, die den Entwurf vorbereiten und ihn auf seinem parlamentarischen Weg begleiten. Eine ihrer wichtigsten, aber auch schwierigsten Aufgaben ist, mit potenziellen Klägern vor oder während des parlamentarischen Verfahrens zu verhandeln.

Spezielle private Agenturen bieten bei der Formulierung des Entwurfs und für das weitere Verfahren ihre Dienste an. Sie vermitteln auch parlamentarische Vertreter an die privaten Initiatoren und dürfen für ihre Dienste Gebühren verlangen. Von besonderer Bedeutung ist, dass entsprechende Initiativen in der Öffentlichkeit, zum Beispiel über Zeitungsanzeigen oder durch die direkte Ansprache potenzieller Gegner des Projekts, publik gemacht werden müssen, um Eingaben beziehungsweise Widersprüche zu ermöglichen. ${ }^{12}$ Diese Phase ist zeitlich streng reglementiert. Die Entwürfe müssen am oder vor dem 27. November beim Parlament eingereicht und vor oder am 11. Dezember öffentlich bekannt gemacht werden. ${ }^{13}$

Der Widerspruch erfolgt in Form einer Petition an das Parlament. Form und Verfahren der Eingabe sind in Standing Orders des House of Commons geregelt. Er kann entweder vom Kläger selbst oder einem beauftragten Dritten oder wiederum von einem Parliamentary Agent eingereicht und vertreten werden. Der Einspruch ist nur zulässig, wenn der Einzelne, die Organisation oder Gemeinde nachweisen kann, dass sie durch die Maßnahme unmittelbar betroffen wäre. Ist dies strittig, entscheidet ein Ausschuss erfahrenerer Hinterbänkler über die Zulässigkeit.

Nach diesem Vorlauf entscheiden der Stellvertreter des Speakers des Unterhauses (Chairman of Ways and Means) und der Vorsitzende der Ausschüsse des Oberhauses (Chairman of Committees), ob der Entwurf zuerst in das Ober- oder Unterhaus kommt. Komplexere Materien und umfangreichere kommunale Initiativen gehen in der Regel zuerst ins House of Lords, solche mit eher politischem Inhalt zuerst ins House of Commons. Wie bei Public Bills ist die Einbringung bereits die erste Lesung. Nach der zweiten Lesung erfolgt die Ausschussberatung. Der Ausschuss hat quasi-gerichtliche Kompetenzen und entscheidet darüber, ob und in welcher Form der Entwurf weiter geleitet wird. Er kann Veränderungen und Ergänzungen vornehmen oder die gesamte Initiative als „Case not proved“ (zuletzt geschehen 1995) beenden. Auch die dritte Lesung mit letzten Debatten und Ergänzungen erfolgt wieder nach einem strengen Zeitplan. Die Lesungen im Oberhaus laufen nach dem gleichen Schema wie im Unterhaus ab. Macht das Oberhaus Ergänzungen, muss das Unterhaus abschließend noch einmal darüber befinden.

Kommunalverwaltungen können im Zuge spezieller Local und Personal Acts aktiv werden, etwa um besondere Kompetenzen und Verwaltungsstrukturen zu erhalten. Solche lokalen Regelungen durch Parlamentsgesetze haben eine lange Tradition und zu einem gewissen "Wildwuchs“ geführt. Im Local Government Act von 1972 wurde schließlich die

11 Vgl. ebenda.

12 Vgl. Thomas Krumm / Thomas Noetzel, Das Regierungssystem Großbritanniens, München 2006, S. 188.

13 Vgl. House of Commons Information Office, a.a.O. S. 2. 
Aufhebung aller dieser alten Regelungen beschlossen, sofern sie nicht durch neue Gesetze bestätigt und zugleich modernisiert werden. ${ }^{14}$

Nach der Verabschiedung des Human Rights Acts 1998 hat das Joint Committee on Human Rights eine Reihe von Ergänzungen zu den Standing Orders des House of Commons vorgeschlagen. Dabei wurden auch die Regelungen zu Private Bills um eine Menschenrechtskomponente ergänzt. Promotors müssen nun eine Erklärung zum Menschenrechtsstatus ihrer Initiative beifügen, und ein Ministerium muss wiederum zu dieser Erklärung Stellung nehmen. ${ }^{15}$

\subsection{Hybrid Bills in Großbritannien}

Einzelinteressen finden aber nicht nur durch Private Bills Eingang in den britischen Gesetzgebungsprozess. Hybrid Bills sind eine Form der Public Bills, die ebenfalls Interessen Einzelner oder von Organisationen inkorporieren. ${ }^{16}$ Sie kommen bei Projekten zum Zuge, die von nationalem Interesse sind, aber einzelne Personen oder lokale Organisationen (zum Beispiel Gemeinden) umfassender betreffen als den Rest des Landes. Dies ist beispielsweise bei größeren Verkehrsprojekten der Fall, während kleinere Vorhaben, häufig im Bereich der Errichtung privater Eisenbahnverbindungen, durch Private Bills geregelt werden. Hybrid Bills werden sehr selten genutzt; in den letzten 20 Jahren wurden lediglich zehn Gesetze dieses Typs verabschiedet. ${ }^{17}$ Gegenwärtig wird eine weitere Hybrid Bill verhandelt, mit der einem Unternehmen („Crossrail“) die Einrichtung einer Schnellbahnlinie ermöglicht werden soll, die Londons Osten und Westen miteinander verbindet (deshalb „Crossrail-Bill“). Sollte das Gesetz Zustimmung finden, wäre dies die 11. Hybrid Bill in den letzten 20 Jahren. Hybrid Bills können zwar von der Regierung und den Abgeordneten des Unterhauses initiiert werden, faktisch können sich aber fast ausschließlich Vorschläge der Regierung durchsetzen. ${ }^{18}$

Nach der ersten Lesung einer jeden Public Bill überprüft der „Clerk of the House“, ob nicht eventuell private Interessen betroffen sind und somit das Verfahren für Hybrid Bills angewandt werden muss. Wenn dies der Fall ist, unterzieht der „Examiner of Petitions for Private Bills“ und ein Geschäftsordnungsausschuss die Gesetzesvorlage (wie bei den Private Bills) einer Prüfung. Wenn eine Übereinstimmung des Entwurfs mit den Statuten für Private Bills besteht, wird das parlamentarische Verfahren für Hybrid Bills angewandt, ansonsten nimmt er als Public Bill seinen Lauf. In der zweiten Lesung einer Hybrid Bill bekommen Personen oder Organisationen, die durch das Gesetz besonders betroffen wären, die

14 Vgl. ebenda, S. 7.

15 Michael Rush, Parliament and Government: An annotated Bibliography of Government Publications for the 2001-02 Parliamentary Session, in: Parliamentary Affairs, 57. Jg. (2004), S. $469-$ 486, S. 470.

16 House of Commons Information Office, Hybrid Bills, Factsheet L5, London 2005.

17 Ebenda, S. 6.

18 Im Gegensatz zu den Private Bills kommt die Gesetzesinitiative also vollständig aus dem Parlament und nicht von außen, weswegen sie auch zu den Public Bills gezählt werden. Initiativen von Abgeordneten der Opposition (Private Members) dienen meist nur dazu Öffentlichkeit zu erhalten, um so ein politisches Statement abzugeben oder die Stimmung für ein Thema zu testen, auch wenn die Initiative keine Aussicht auf Erfolg hat. 
Möglichkeit in einem Select Committee ihre Position zu vertreten, Einsprüche zu formulieren und Zeugen zu benennen. ${ }^{19}$ Sodann berichtet das Select Committee dem Plenum des Unterhauses und schlägt Änderungen vor. Liegen keine Petitionen gegen den Gesetzentwurf vor, wird die Select Committee-Phase übersprungen und der Entwurf direkt in einem Standing Committee oder dem Committee of the Whole House (Plenum) weiter behandelt. Nach der dritten Lesung werden Hybrid Bills wie Public Bills an das Oberhaus überwiesen, wo sich Betroffene im Rahmen eines Select Committee erneut einbringen können und angehört werden. Zusammenfassend gesagt vereinigen Hybrid Bills also Merkmale von Public Bills und Private Bills. Der Anstoß zu einem Gesetzentwurf kommt wie bei den Public Bills aus dem Parlament. Anders als bei reinen Public Bills werden im parlamentarischen Prozess jedoch die Betroffenen wie beim Private Bill-Verfahren angehört und miteinbezogen.

\subsection{Schottland}

Im Zuge der exekutiven Devolution des Zentralstaates ist auch im Schottischen Parlament ein Initiativrecht für Private Bills eingeführt worden. Nach Regel 9A.1.1 der Standing Orders werden Private Bills ,introduced for the purpose of obtaining for an individual person, body corporate or unincorporated association of persons (,the promoter') particular powers or benefits in excess of, or in conflict with, the general law, and includes a Bill relating to the estate, property, status or style, or otherwise relating to the personal affairs, of the promoter" 20 .

Private Bills, die sich ausschließlich mit im Zuge der Devolution übertragenen Kompetenzen befassen, dürfen nur ins Schottische Parlament eingebracht werden. Private Bills, die nicht-devoluierte schottische Angelegenheiten betreffen („reserved matters“, vgl. Abschnitt 5 des Scotland Acts von 1998), müssen weiterhin nach dem System des Private Legislation Procedure (Scotland) Act von 1936 und den General Orders des Parlaments in Westminster behandelt werden. ${ }^{21}$ Während es im Londoner Unterhaus ein enges Zeitfenster Ende November gibt, können in Schottland Private Bills an jedem Sitzungstag eingebracht werden. ${ }^{22}$

Insgesamt lässt sich wie in Westminster ein dreistufiger Prozess für Private Bills skizzieren. Der erste formale Schritt, die „Preliminary Stage“, besteht in der Einbringung des Entwurfs sowie der erforderlichen Zusatzdokumente und der Einrichtung eines Private Bill-Ausschusses mit drei bis fünf Mitgliedern durch eine Parlamentsresolution. Die Zusatzdokumente bestehen aus Explanatory Notes, in denen in allgemeinverständlicher Sprache der Zweck der einzelnen Normen erklärt wird, einem Promoters Memorandum, in dem die Policy-Ziele noch einmal erklärt werden und warum man diese Ziele nicht auf

19 Wie die Gegner einer Private Bill müssen auch sie zuvor eine Petition innerhalb einer Frist an das Private Bill Office gerichtet haben.

20 Vgl. www.scottish.parliament.uk/business/bills/privateBills.htm, 6. März 2006. Anders als in Schottland kennt die Walisische Versammlung mit ihren lediglich sekundären Gesetzgebungskompetenzen kein privates Initiativrecht.

21 Vgl. www.scottish.parliament.uk/business/bills/billguidance/gprb-c.htm, 6. März 2006.

22 Mit der Einbringung muss der „Promoter“ (oder Agent) auch eine Gebühr entrichten. 
alternativen Wegen erreichen kann und welche Konsultationen in dieser Sache bisher unternommen worden sind, sowie einem Promoters Statement, in dem alle bisherigen Aktivitäten des Promoters im Zusammenhang mit der Einbringung, wie zum Beispiel Konsensgespräche mit Gegnern oder Zeitungsanzeigen, dokumentiert sind. ${ }^{23}$

Im Frühjahr 2006 gab es insgesamt vier Parlamentsausschüsse für Private Bills in Schottland; zwei davon haben sich mit Straßenbahn-Initiativen in Edinburgh befasst, zwei mit dem Ausbau von Eisenbahnstrecken. ${ }^{24}$ Die Ausschüsse müssen sich aus Angehörigen verschiedener Parteien zusammensetzen, und aufgrund ihres quasi-gerichtlichen Charakters müssen ihre Mitglieder als neutral und unparteilich in der zu regulierenden Materie gelten, dürfen zum Beispiel nicht aus der Region kommen, die von dem Vorhaben betroffen ist oder durch die Maßnahme finanzielle Vorteile erhält. Der Ausschuss erstellt einen Bericht, in dem er dem Parlament empfiehlt, ob dem Anliegen grundsätzlich zuzustimmen ist oder nicht und ob die Initiative als Private Bill fortgeführt werden soll. Erst danach wird ein Termin für die parlamentarische „Preliminary Stage“-Debatte angesetzt. Nach Regel 9A.6.1 können Personen, Körperschaften oder auch nichteingetragene Vereine (unincorporated association) einen Einspruch zu einer Private Bill hinterlegen, die eigene Interessen nachteilig betreffen könnte. Solche Petitionen müssen bei der Parlamentsverwaltung innerhalb einer Einspruchsfrist von 60 Tagen nach Einbringung der Initiative in das Parlament eingereicht werden. In der zweiten Stufe, der „Consideration Stage“, werden die Details des Entwurfs sowie die öffentlichen Einwände und Ergänzungen beraten. Schließlich wird auf der „Final Stage“ nach Abschluss der Beratungen über letzte Ergänzungen des Wortlauts („Amendments“) abgestimmt, ehe der Entwurf durch „Royal Assent“ der Königin zum Gesetz wird. Das schottische Verfahren der Private Bills unterscheidet sich vom britischen durch eine stärkere Gewichtung der ersten Phase und einer hohen Regulierungsdichte durch die Standing Orders.

\section{USA und Kanada}

Wie in Großbritannien ist in den USA ein starker Rückgang bei der Einbringung von Private Bills in den Kongress zu beobachten. Während zwischen 1817 und 1971 in jeder Kongressperiode Hunderte von Private Bills behandelt wurden, ist deren Zahl, nicht zuletzt unter dem Eindruck einer Reihe von Bestechungsskandalen, in den letzten Perioden auf unter 30 gesunken. ${ }^{25}$ Im 103. Kongress ab 1993 gab es lediglich acht Private Laws, während noch im 59. Kongress (1905) 90 Prozent (6248), im 82. Kongress (1951) 63 Prozent aller Gesetze Private Laws waren. ${ }^{26}$ Was ein Gesetz als privat kennzeichnet, ist nirgends

23 Für bestimmte Sachgebiete kommen weitere Dokumente hinzu, wie zum Beispiel Karten und Pläne, Umweltgutachten oder Finanzierungspläne, vgl. www.scottish.parliament.uk/business/ bills/privateBills.htm, 6. März 2006.

24 Insgesamt wurden seit Einführung 1999 acht Private Bills in Ausschüssen beraten, weitere Themen waren zum Beispiel die Errichtung eines Offshore Windparks und die Reorganisation der National Galeries.

25 Richard S. Beth, Private Bills: Procedure in the House. CRS Report for Congress, Order Code 98-628 GOV, Washington 2004 (www.rules.house.gov/archives/98-628.pdf, 1. März 2006).

26 Richard T. Boylan, Private bills: A theoretical and empirical study of lobbying, in: Public Choice III, 2002, S. $19-47$, S. 20. 
niedergelegt. In der Regel erkennt man sie an ihrem Titel mit der Formulierung „For the benefit of...". Private Laws sind oft der letzte Ausweg für Individuen oder Organisationen, wenn alle administrativen und gerichtlichen Mittel ausgeschöpft sind. Verboten sind Initiativen im Bereich des Pensionswesens, des Brückenbaus und militärischer Archive. Das Auftreten eines Co-Sponsors neben dem Hauptsponsoren ist nicht zulässig. ${ }^{27}$ Viele der gegenwärtig verhandelten Initiativen betreffen Entscheidungen der Judikative, etwa bei Immigrationsfragen wie Aufenthaltsstatus oder Visaklassifikation, aber auch Patente und Urheberrechte sowie inländische und ausländische Leistungsansprüche an die Regierung. Auch Steuerfragen, öffentliches Land und Veteranenunterstützung sind Gegenstand von Private Laws geworden. ${ }^{28}$

Wie in Großbritannien wurde eine Reihe von Sachbereichen, die üblicherweise Anlass für Private Bills waren, nun auf die administrative Ebene verschoben. Diese Entwicklung wird unter anderem darauf zurückgeführt, dass der Kongress der Verwaltung mehr Autorität beziehungsweise Entscheidungskompetenz zugebilligt hat. Als eine zweite Erklärung für den Rückgang werden strengere Public Laws in diesen Feldern angeführt. ${ }^{29}$ Des Weiteren werden vermehrte Korruptionsskandale für die sinkenden Zahlen verantwortlich gemacht. Danach seien Kongressmitglieder vorsichtiger bei der Übernahme einer Private Bill geworden, um nicht in den Verdacht der Bestechlichkeit zu geraten. So waren zum Beispiel lange Zeit Private Bills zum Aufschub einer Ausweisung von Immigranten bei laufenden Verfahren üblich. „While many such private bills were introduced by members of Congress aiming to pursue electoral or humanitarian objectives, many such private bills were introduced in exchange for bribes. " 30 Seit die Immigrationsbehörde solche Aufschübe selbst entscheiden kann, ist die Anzahl der Private Bills für Immigranten drastisch gesunken. Da erst danach ein signifikanter Anstieg von Korruptionsfällen zu beobachten war, können diese nicht den Rückgang der Private Bills verursacht haben.

In den USA lässt sich langfristig eine starke zyklische Aktivität bei Private Bills beobachten, allerdings mit einer bis heute anhaltenden deutlich rückläufigen Tendenz seit den 1970er Jahren. Richard T. Boylan führt diese Schwankungen auf die Dialektik des Verhaltens von Kongressmitgliedern zurück, die das Einbringen von Private Bills einerseits als Service für ihren Wahlkreis oder als Gegenleistung für Parteispenden betreiben, andererseits aber in der Öffentlichkeit Korruptions- und Bestechungsvorwürfe riskieren, die die Wiederwahlchancen beeinträchtigen können. Damit kann erklärt werden, warum nach Korruptionsfällen die Nutzung von Private Bills sinkt, nicht aber, warum es zu einem säkularen Rückgang gekommen ist. Dies erklärt Boylan damit, dass in Nach-Skandal-Phasen die Bereitschaft des Kongresses insgesamt, entsprechende Kompetenzen nicht selbst auszuüben, sondern durch Public Laws an die Verwaltung zu delegieren, besonders hoch ist. Auf diese Weise soll quasi eine Selbstreinigung bewirkt werden, durch die das Ansehen und die Autorität des Kongresses wieder erhöht wird. In Phasen, in denen das Ansehen des Kongresses hoch ist, ist es dann für einzelne Mitglieder wieder einfacher, private Legislation ohne erhöhtes Risiko eines Skandals anzunehmen. ${ }^{31}$

27 Richard S. Beth, a.a.O.

28 Richard T. Boylan, a.a.O., S. 20.

$29 \mathrm{Vgl}$. ebenda.

30 Vgl. ebenda, S. 21.

31 Vgl. ebenda, S. 30. 
In Kanada gilt, analog zu Private Members' (beziehungsweise Senators') Bills, dass Private Bills sowohl im House of Commons als auch im Senat eingebracht werden können. Tatsächlich werden Private Bills aber fast ausschließlich im Senat eingebracht. ${ }^{32} \mathrm{Wie}$ in den anderen Ländern üblich, müssen die Begünstigten im Gesetz namentlich genannt werden, und es muss in öffentlichen Anzeigen auf die geplante Maßnahme hingewiesen werden. Die Anzeigen müssen über einen Zeitraum von vier Wochen wöchentlich in Englisch und Französisch geschaltet werden, sofern die Zusammensetzung der Bevölkerung in der Region dies erfordert. ${ }^{33}$ Beim Einreichen des Entwurfs im Senat in englisch oder französisch muss zugleich eine Gebühr für die Übersetzung in die jeweils andere Sprache, den Druck von Kopien und die spätere Veröffentlichung im Gesetzesblatt sowie eine Bearbeitungsgebühr von \$ 200 bezahlt werden (Rule 110). Bei der ersten Lesung im Senat kann der Entwurf auf Antrag von zwei Senatoren an den Ständigen Ausschuss für Verfassungsfragen überwiesen werden, falls es Zweifel gibt, ob die Materie nicht in den ausschließlichen Zuständigkeitsbereich der Provinzen fällt. Eine Besonderheit des kanadischen Verfahrens ist, dass der Senat den Entwurf jederzeit zur Überprüfung an den Supreme Court überweisen kann (Rule 112). Petitionen gegen den Entwurf werden zunächst von einem „Examiner“ auf ihre Zulässigkeit überprüft und später schwerpunktmäßig in der Ausschussphase beraten.

Wie in Großbritannien und den USA sind Private Bills in Kanada in den letzten Jahren rückläufig, wenn auch moderater. Gegenstände von Private Bills waren neben der bereits erwähnten Möglichkeit der Ehescheidung vor allem Einzelfallregelungen im Bereich der Banken, der Versicherungen und des Kreditgewerbes, der öffentlichen Infrastruktur, von Handelskammern und religiösen sowie karitativen Organisationen. ${ }^{34}$ Lag ihre Zahl in den 1990er Jahren relativ konstant bei etwa drei Gesetzen pro Jahr, so ist seit 2002 nur noch ein Gesetz pro Jahr zu verzeichnen. 2003 wurden per Gesetz Verbände von Finanz- und Versicherungsdienstleistern fusioniert, 2004 ein theologisches College und 2005 die Generalsynode der Anglikanischen Kirche von Kanada reorganisiert. ${ }^{35}$

\section{Deutschland}

In der Bundesrepublik Deutschland sind Einzelfallgesetze teilweise mit den angelsächsischen Private Bills vergleichbar. Bei diesen kommt allerdings das Element des privaten Initiativrechts beziehungsweise des „Gespanns" von außerparlamentarischem Klient und parlamentarischem Promotor hinzu. Gravierender Unterschied ist also, dass Einzelfallgesetze in der Bundesrepublik nicht von einzelnen Abgeordneten eingebracht werden können. Dennoch scheint aufgrund einer partiellen Affinität beider Rechtsinstitute ein Ausblick auf die bundesdeutsche Regelung sinnvoll, akzentuiert sie doch die unterschiedlichen Rechtsund Politikverständnisse. Verfassungssystematisch erinnert der in den USA praktizierte Einsatz von Private Bills zur Revision justizieller Entscheidungen an die Praxis des Macht-

$32 \mathrm{Zu}$ den Verfahrensregeln vgl. www.parl.gc.ca/information/about/process/senate/rules-e/senrules_ 11-e.htm, 6. März 2006.

33 Ebenda.

34 Vgl. http://laws.justice.gc.ca/en/privlaw/index.html, 1. Dezember 2006.

35 www.parl.gc.ca, 6. März 2006. 
spruchs im Deutschland des 18. Jahrhunderts, wie er etwa im Machtspruch Friedrichs II. im so genannten Müller-Arnold-Fall Verfassungsgeschichte geschrieben hat. ${ }^{36}$ Private Bills, die auf (verwaltungs)gerichtliche Entscheidungen Bezug nehmen und diese revidieren, sind als Eingriff der Legislative in den Kompetenzbereich der Judikative in gewisser Weise eine moderne Form des „Machtspruchs“.

In der Bundesrepublik sind Einzelfallgesetze nicht grundsätzlich verboten, sondern nach Art. 19 Abs. 1 S. 1 GG lediglich dann, wenn sie Grundrechte einschränken. ${ }^{37}$ Eine weitere Einschränkung ergibt sich dadurch, dass Gesetze grundsätzlich abstrakt-generell sein, also für eine unbestimmte Anzahl von Sachverhalten und Personen gelten müssen. Die individuelle (bestimmter Personenkreis) und konkrete (Geltung für bestimmte Fälle) Ausgestaltung erfolgt dann auf der Ebene von Verwaltungsakten, typischerweise als Einzelfallregelung nach $\$ 35$ S. 1 VwVfG. Erst auf Verwaltungsebene wird also der individuelle Personenkreis und die konkrete Fall- beziehungsweise Sachgeltung bestimmt. Es erfolgt damit im Öffentlichen Recht eine Zuweisung individuell-konkreter Maßnahmen an den Kompetenzbereich der vollziehenden Verwaltung und abstrakt-genereller Regelungen an den des Gesetzgebers.

Allerdings wird diese Trennung, mit der der Gewaltenteilung und dem Rechtsstaatsprinzip Rechnung getragen werden soll, nicht streng durchgehalten. So ist etwa die Verwaltung im Bereich der Normsetzung durch Rechtsverordnungen, Satzungen und Verwaltungsvorschriften tätig, und umgekehrt sind dem Gesetzgeber Einzelfallmaßnahmen nicht generell verboten, bedürfen jedoch nach Auffassung des Bundesverfassungsgerichts besonderer Rechtfertigung. Ein Gesetzesbegriff, der lediglich allgemeine Regelungen als Inhalte zulasse, lässt sich laut BVerfG nicht aus dem Rechtsstaatsprinzip ableiten. ${ }^{38}$

Die Anwendung von Einzelfall- und Maßnahmengesetzen gerade im Bereich der Verkehrsinfrastruktur wurde in den 1970er Jahren beim Ausbau des Bundesfernstraßennetzes und dann wieder in den 1990er Jahren relevant, als für eine Reihe von „Verkehrsprojekten Deutsche Einheit" die Gesetzesform (statt der Verwaltungsakte des Planfeststellungsverfahrens) gewählt wurde, um die Planung zu beschleunigen. So wurde zum Beispiel durch Gesetzesbeschluss die „Südumfahrung Stendal“ als Teilstück der neuen Eisenbahn-Hochgeschwindigkeitsstrecke Hannover - Berlin zugelassen, wogegen von Anwohnern geklagt wurde. Mit dem Hinweis, auch Detailpläne seien einer gesetzlichen Regelung zugänglich, hat das BVerfG das Gesetz zugelassen. ${ }^{39}$ Solche Detailpläne gehörten nicht zum Kernbereich exekutiver Eigenverantwortung. Bei guten Gründen, wie sie das Gericht im Falle der Beschleunigung des Aufbaus der Infrastruktur in den neuen Ländern für gegeben sah, dürfe das Parlament solche Entscheidungen durch Gesetz an sich ziehen.

36 Vgl. Fn. 4.

37 Vgl. BVerfGE 25, S. 371 („lex Rheinstahl“).

38 Vgl. BVerfGE 25, S. 96: „Ein über Art. 19 Abs. 1 GG hinausgreifendes Verbot von Einzelfallgesetzen lässt sich insbesondere nicht aus dem Rechtsstaatsprinzip herleiten. Dem Grundgesetz kann nicht entnommen werden, dass es - von Art. 19 Abs. 1 GG abgesehen - von einem Gesetzesbegriff ausgeht, der als Inhalt der Gesetze lediglich generelle Regelungen zulässt. Auch die gesetzliche Regelung eines einzelnen Falles kann erforderlich sein. Das gilt vor allem im Bereich der Wirtschafts- und Sozialordnung. Mit der Regelung eines einzelnen Falles greift der Gesetzgeber nicht notwendig in die Funktionen ein, die die Verfassung der vollziehenden Gewalt oder der Rechtsprechung vorbehalten hat."

39 Vgl. BVerfGE 95, S. 1, S. 14 ff. 


\section{Legitimitätsressource oder „undifferentiated polity“?}

Die Bezeichnung Private Bill darf nicht darüber hinwegtäuschen, dass der Prozess vollständig unter parlamentarischer Kontrolle steht, was durch das „Gespann“ von privatem Initiator und parlamentarischen Agent ermöglicht wird. Die Praxis privater Gesetzesinitiativen ist deshalb nicht als (illegitimes) Lobbying oder als Privatisierung der Gesetzgebungsaktivität zu begreifen, auch wenn sie in den Vereinigten Staaten bis zu ihrem Niedergang in den 1970er Jahren stärker klientelistische Züge aufwies. Vielmehr deuten die langfristige grundsätzliche Bewährung dieses Verfahrens und seine Einführung im Schottischen Parlament darauf hin, dass mit diesem Instrument eine zusätzliche Legitimitätsressource erschlossen wird. In diesem Sinne sind Private Bills eine der Komponenten, die für die Stabilität des Westminster-Modells gesorgt haben. Private Bills erhöhen die Responsivität des Parlaments, weil sie es für einen „Querimport“ von privaten beziehungsweise partikularen Interessen öffnen, ohne die Souveränität des Parlaments zu verletzen. Sie relativieren damit die Rigorosität der ausschließlichen Verpflichtung des Gesetzgebers auf abstrakt-generelle Gesetze, die ja auch in der Bundesrepublik nicht konsequent durchgehalten wird. Demgegenüber kann die angelsächsische Unterscheidung von Public und Private Bills (mit der britischen Mischform der Hybrid Bills) in diesem Feld der Einzelfallgesetze mehr Transparenz gewährleisten.

Im Vergleich zur administrativen Regelung erhöht die Beteiligung des Parlaments durch Private Bills zunächst die demokratische Legitimität der Entscheidungen. Dies darf aber nicht dazu führen, dass das Parlament permanent mit der Regelung von Detail- und Sonderinteressen befasst wird, die rein administrativ wahrscheinlich effizienter zu handhaben sind. Die rückläufige Zahl der Private Bills verweist darauf, dass die Dimension der Legitimitätssteigerung bei einer Detailentscheidung durch Parlamentsbeteiligung nicht dominant sein kann. Vielmehr stellt sie einen von mehreren Aspekten dar, die die unterschiedlichen Politischen und Parlamentskulturen in angelsächsischen und kontinentaleuropäischen Staaten prägen. Während bei den britischen und amerikanischen Private Bills der funktionale Schwerpunkt auf Leistungsrechten liegt, betont in der Bundesrepublik Art. 3 in Verbindung mit Art. 19 Abs. 1 S. 1 GG den Abwehraspekt und die funktionale Gewaltenteilung zwischen Gesetzgeber und Verwaltung. Der Unterschied von Abwehr- und Leistungsrechten wird in der US-amerikanischen Formulierung „For the benefit of ... “ besonders deutlich. Eine solche namentliche individuelle Adressierung eines Gesetzes ist mit deutschem Verfassungsrecht nicht vereinbar, da hier der Adressatenkreis förmlicher Gesetze abstrakt-generell bezeichnet werden muss. Der als „decline of private legislation “ 40 zu beschreibende Rückgang in Großbritannien wie auch in den USA und Kanada lässt sich als eine Annäherung an das (bundesdeutsche) Modell funktionaler Gewaltenteilung verstehen, bei der konkret-individuelle Maßnahmen Angelegenheit der Verwaltung und nicht des Gesetzgebers sind. Damit lässt er sich in den Kontext der Diskussion um den Niedergang des Westminster-Modells einordnen. ${ }^{41}$

40 Vgl. Jeffrey S. Hill / Kenneth C. Williams, The decline of Private Bills - resource allocation, credit claiming, and the decision to delegate, in: American Journal of Political Science, 37. Jg. (1993), S. $1008-1031$.

41 Vgl. zum Beispiel Roland Sturm, Regieren in Großbritannien. Die aktuelle britische Debatte zum Verständnis des Westminster-Modells, in: ZParl, 37. Jg. (2006), S. 795 - 811. 